\title{
A Mortar Element Method for Coupling Natural Boundary Element Method and Finite Element Method for Unbounded Domain Problem
}

\author{
Sheng Zhang and Dehao Yu
}

\begin{abstract}
In this paper, a mortar element method is presented for coupling natural boundary element method and finite element method for the Dirichlet exterior problem. Optimal error estimate is obtained and some numerical results are reported.
\end{abstract}

\section{Introduction}

In many fields of scientific and engineering computing, it is necessary to solve boundary values problems of partial differential equations over unbounded domains. For this kind of problems, the standard techniques such as the finite element method, which is effective for most problems over bounded domain, will meet some difficulties and the corresponding computing cost will be very high. As a alternative, boundary element method is considered and developed for this kind of problems and great progress has been made in this field. Also, boundary element method has its own weakness (for example, we will meet some difficulties when treating complicated bound domain problems and so on). So, it seems that the coupling method of boundary element method and finite element method which combines the advantages of boundary element method with those of finite element method may be more attractive for this kind of problems. Many authors have made contributions to the coupling method of this kind (refer to [11,12,15,17-21,23-26,28,29,32,35,36,39,40] and references therein) and research in this direction is of great importance both in theory and practical computation.

Generally, the coupling method of this kind can be described as follows. First, a artificial boundary is introduced which divide the unbounded domain into two subdomains - a bounded inner one and an unbounded outer one. Then we couple the finite element method which is used for the bounded inner one and boundary element method which is used for the unbounded outer one together.

1991 Mathematics Subject Classification. 65N30, 65N38, 65N15.

Key words and phrases. Mortar element method, Finite element method, Boundary element method, Error estimate, Unbounded domain problem.

This work is supported by the Special Funds of State Major Basic Research Projects of China (G19990328) and the Knowledge Innovation Program of Chinese Academy of Science. 
There are many kinds of methods to archive this kind of coupling and mortar element method is one of them. Compared with other coupling method, the mortar element method appears to be attractive because meshes on different subdomains need not align across subdomain interface in this method which provide us a lot of flexibility to triangulation subdomains independently of each other and the matching of discretizations on subdomains is only enforced weakly. We refer to $[5-8,16,27,30,31,33]$ and references therein for mortar element method and some applications for detail. The purpose of this paper is to investigate a mortar element method for this kind of coupling and to obtain the corresponding error estimate.

Another feature of this paper we want to mention is that natural boundary reduction method is used. Natural boundary reduction method, which is also known as the exact artificial boundary condition method, is suggested and developed first by K. Feng, H. Han and D. Yu in the early eighties of twenty century (refer to $[17-19,24,25,35])$. And a very similar method, the so-called DtN method, has also been devised by J. B. Keller and D. Givoli in the late eighties of twenty century (refer to $[21,29]$ ). Compared with many other approaches of reduction, natural boundary reduction method has its own advantages(refer to [19,36,39] for detail).

The remainder of this paper is organized as follows: In section 2, we describe our model problem and introduce natural boundary reduction method and mortar element method. The corresponding error estimate is presented in section 3. In section 4 , some numerical results are shown.

\section{Model problem and discretization}

We adopt the notations for Sobolev space, their norms and semi-norms as presented in [10,14]. Let $\Omega$ be a Lipschitz bounded domain in $\mathbb{R}^{2}, \Omega^{c}=\mathbb{R}^{2} \backslash(\Omega \cup \partial \Omega)$, $f \in L^{2}\left(\Omega^{c}\right)$ be a given compactly supported function. We consider the following model problem

$$
\left\{\begin{aligned}
-\triangle u & =f, \quad \text { in } \Omega^{c}, \\
u & =0, \quad \text { on } \partial \Omega,
\end{aligned}\right.
$$

subject to the asymptotic conditions

$$
u(x, y)=\alpha+O(1 / r), \quad|\nabla u(x, y)|=O\left(1 / r^{2}\right),
$$

as $r=\sqrt{x^{2}+y^{2}} \rightarrow \infty$ where $\alpha$ is a constant. Define

$$
H_{\triangle}^{1}\left(\Omega^{c}\right)=\left\{v\left|\frac{v}{\sqrt{r^{2}+1} \ln \left(r^{2}+2\right)}, \frac{\partial v}{\partial x}, \frac{\partial v}{\partial y} \in L^{2}\left(\Omega^{c}\right), v\right|_{\partial \Omega}=0\right\}
$$

and

$$
a(w, v)=\iint_{\Omega^{c}} \nabla w \cdot \nabla v d x d y, \quad \forall w, v \in H_{\triangle}^{1}\left(\Omega^{c}\right) .
$$

Then the corresponding variational form of (2.1) can be written as: Find $u \in$ $H_{\triangle}^{1}\left(\Omega^{c}\right)$ such that

$$
a(u, v)=(f, v), \quad \forall v \in H_{\triangle}^{1}\left(\Omega^{c}\right) .
$$

Due to the hypothesis on $f$, we can choose a circle disc $\Omega_{0}$ containing $\bar{\Omega}$ and supp $f$. Let $\Omega_{1}=\Omega^{c} \cap \Omega_{0}, \Omega_{2}=\Omega_{0}^{c}=\mathbb{R}^{2} \backslash\left(\Omega_{0} \cup \partial \Omega_{0}\right)$ and $\Gamma=\partial \Omega_{0}$ (see Figure 1$)$. Then we have

$$
a(u, v)=a_{1}(u, v)+a_{2}(u, v)
$$




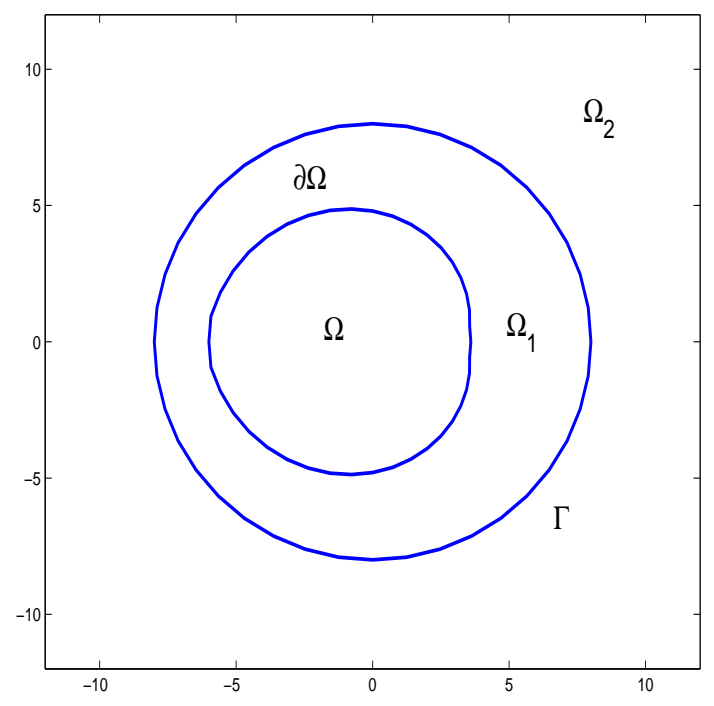

Figure 1. Artificial boundary

where $a_{i}(u, v)=\iint_{\Omega_{i}} \nabla u \cdot \nabla v d x d y, \quad i=1,2$.

Next, we introduce the natural boundary reduction method. Let $u$ be the solution of problem (2.1). From Green's formula on $\Omega_{2}$, we have

$$
a_{2}(u, v)=\int_{\Gamma} \frac{\partial}{\partial n} u(z) \cdot v(z) d z+\iint_{\Omega_{2}} f v d x d y .
$$

Let $G\left(z, z^{\prime}\right)$ be the Green's function for the Laplace operator on the domain $\Omega_{2}$, which satisfies

$$
\left\{\begin{aligned}
-\triangle G\left(z, z^{\prime}\right) & =\delta\left(z-z^{\prime}\right), & \forall z, z^{\prime} \in \Omega_{2}, \\
\left.G\left(z, z^{\prime}\right)\right|_{z \in \Gamma} & =0, & \forall z^{\prime} \in \Omega_{2},
\end{aligned}\right.
$$

subject to the same asymptotic conditions as $u$. By taking $w=G\left(z, z^{\prime}\right), v=u$ in Green's second formula

$$
\iint_{\Omega_{2}}(w \triangle v-v \triangle w) d z^{\prime}=\int_{\Gamma}\left(w \frac{\partial v}{\partial n^{\prime}}-v \frac{\partial w}{\partial n^{\prime}}\right) d z^{\prime},
$$

we obtain (see, $[36,39]$ )

$$
u(z)=\iint_{\Omega_{2}} f\left(z^{\prime}\right) G\left(z, z^{\prime}\right) d z^{\prime}-\int_{\Gamma} \frac{\partial}{\partial n^{\prime}} G\left(z, z^{\prime}\right) u\left(z^{\prime}\right) d z^{\prime}, \quad \forall z \in \Omega_{2} .
$$

Thus we obtain

$$
\frac{\partial u}{\partial n}(z)=\iint_{\Omega_{2}} f\left(z^{\prime}\right) \frac{\partial}{\partial n} G\left(z, z^{\prime}\right) d z^{\prime}-\int_{\Gamma} \frac{\partial^{2}}{\partial n \partial n^{\prime}} G\left(z, z^{\prime}\right) u\left(z^{\prime}\right) d z^{\prime}, \quad \forall z \in \Gamma,
$$

where $n$ and $n^{\prime}$ denote the exterior normal vectors on $\Gamma$ (viewed as the boundary of $\Omega_{2}$ ) at the respective points $z$ and $z^{\prime}$. Let

$$
K u(z)=-\int_{\Gamma} \frac{\partial^{2}}{\partial n \partial n^{\prime}} G\left(z, z^{\prime}\right) u\left(z^{\prime}\right) d z^{\prime}, \quad z \in \Gamma .
$$


Then, it follows from (2.4), (2.5), (2.6) and the fact that supp $f \subset \Omega_{0}$ that

$$
a_{2}(u, v)=\int_{\Gamma} K u(z) \cdot v(z) d z
$$

Define $H_{*}^{1}\left(\Omega_{1}\right)=\left\{v\left|\quad v \in H^{1}\left(\Omega_{1}\right), v\right|_{\partial \Omega}=0\right\}$ and

$$
b(u, v)=a_{1}(u, v)+<K u, v>_{\Gamma},
$$

where $\left\langle\cdot, \cdot>_{\Gamma}\right.$ denotes the $L^{2}$ inner product on $\Gamma$. With $(2.3)$ and $(2.7)$, we can rewrite the variational form $(2.2)$ as:

Find $u \in H_{*}^{1}\left(\Omega_{1}\right)$ such that

$$
b(u, v)=\iint_{\Omega_{1}} f v d x d y, \quad \forall v \in H_{*}^{1}\left(\Omega_{1}\right) .
$$

REMARK 2.1. The operator $K: H^{\frac{1}{2}}(\Gamma) \mapsto H^{-\frac{1}{2}}(\Gamma)$ is just the DirichletNeumann operator (Steklov-Poincaré operator) for $\Omega_{2}$ (refer to [37]). So, it is symmetric and semi-positive definite with respect to the inner product $\langle\cdot, \cdot\rangle_{\Gamma}$ (refer to $[36,39]$ ), which indicates that $b(\cdot, \cdot)$ is symmetric, bounded and coercive in $H_{*}^{1}\left(\Omega_{1}\right)$. Thus, it follows from the well known Lax-Milgram Theorem that the variational problem (2.9) has unique solution $u \in H_{*}^{1}\left(\Omega_{1}\right)$.

REMARK 2.2. As $\Gamma$ is a circle, the Green's function $G\left(z, z^{\prime}\right)$ can be expressed explicitly. For example, in the case that the center of the circle $\Gamma$ is the origin and its radius is $R$,

$$
\begin{aligned}
& G\left(z, z^{\prime}\right)=\frac{1}{4 \pi} \ln \frac{R^{4}+r^{2} r^{2}-2 R^{2} r r^{\prime} \cos \left(\theta-\theta^{\prime}\right)}{R^{2}\left(r^{2}+r^{\prime 2}-2 r r^{\prime} \cos \left(\theta-\theta^{\prime}\right)\right)}, \\
& z=(r, \theta), \quad z^{\prime}=\left(r^{\prime}, \theta^{\prime}\right) \in \Omega_{2} .
\end{aligned}
$$

Moreover, we have (refer to $[36,39]$ )

$$
\frac{\partial^{2}}{\partial n \partial n^{\prime}} G\left(z, z^{\prime}\right)=\frac{1}{4 \pi \sin ^{2}\left(\left(\theta-\theta^{\prime}\right) / 2\right)}, \quad z=(r, \theta), \quad z^{\prime}=\left(r^{\prime}, \theta^{\prime}\right) \in \Gamma .
$$

It is obvious that these explicit expressions ensure the practical use of the natural boundary reduction method. And these expressions also imply an important advantage of the natural boundary reduction method compared with many other approaches: we need not to solve any boundary integral equation associated with the unbounded subdomain and instead only calculation of certain singular integrations is needed.

REMARK 2.3. To calculate the singular integrations involved, we divide the artificial boundary $\Gamma$ into $m$ circular arcs with the same length. Let $\left\{\phi_{i}\right\}_{i=1}^{m}$ be the set of the nodal basis functions on $\Gamma$. Then we can obtain (see, e.g., $[36,39]$ )

$$
\begin{aligned}
<K \phi_{i}, \phi_{j}>_{\Gamma} & =-\frac{1}{4 \pi} \int_{0}^{2 \pi} \int_{0}^{2 \pi} \frac{\phi_{i}(\theta) \phi_{j}\left(\theta^{\prime}\right)}{\sin ^{2}\left(\left(\theta-\theta^{\prime}\right) / 2\right)} d \theta d \theta^{\prime} \\
& =\frac{4 m^{2}}{\pi^{3}} \sum_{k=1}^{\infty} \frac{1}{k^{3}} \sin ^{4} \frac{k \pi}{m} \cos \frac{2 k(i-j) \pi}{m}, \quad i, j=1, \cdots, m .
\end{aligned}
$$

From this expression, we can easily find that the stiffness matrix of $K$ is symmetric and circulant, which also allows for more efficiency and implies only small memory storage for such stiffness matrix. Moreover, since the series converges quickly, suitable short finite sum can be used to simplify the calculation. 
To derive the mortar element method, we first introduce two triangulations. For $\Omega_{1}$, we choose $m_{1}$ points on $\Gamma: t_{1}^{1}, t_{2}^{1}, \cdots, t_{m_{1}}^{1}$. Then $\Omega_{1}$ is divided into some regular quasi-uniform triangles and curved triangles with diameter $h_{1}$ such that the nodes on $\Gamma$ coincide with the $m_{1}$ points chosen (The usual technique of finite element method in treating curved boundary can be used for the inner boundary of $\Omega_{1}$, i.e. $\partial \Omega$, in the case $\Omega$ has curved boundary. For example, we can use curved element or use some polygon approximating the curved boundary for simplicity and so on. With these techniques, the boundary condition on $\partial \Omega$ is ensured). This triangulation of $\Omega_{1}$ is denoted as $\mathcal{T}_{1}$. Furthermore, we choose another set of points on $\Gamma: t_{1}^{2}, t_{2}^{2}, \cdots, t_{m_{2}}^{2}$ such that $\Gamma$ is divided into $m_{2}$ circular arcs with the same length $h_{2}$ which forms a triangulation denoted by $\mathcal{T}_{2}$. Let $e_{i}^{1}, 1 \leq i \leq m_{1}$ and $e_{j}^{2}, 1 \leq j \leq m_{2}$ denote the curved segment on $\Gamma$ with two endpoints $t_{i}^{1}, t_{i+1}^{1}$ and $t_{j}^{2}, t_{j+1}^{2}$ respectively. Then two independent triangulations of $\Gamma$ can be expressed as $\Gamma_{h 1}=\left\{e_{i}^{1}\right\}_{i=1}^{m_{1}}$ and $\Gamma_{h 2}=\left\{e_{j}^{2}\right\}_{j=1}^{m_{2}}$. For the convenience of presentation, we still need some notations.

Let

$$
\begin{gathered}
W_{1}=\left\{v\left|v \in C^{0}\left(\bar{\Omega}_{1}\right) ; \quad v\right|_{\tau} \in P_{1}(\tau), \quad \forall \tau \in \mathcal{T}_{1},\left.\quad v\right|_{\partial \Omega}=0\right\}, \\
U_{i}=\left\{v\left|v \in C^{0}(\Gamma) ; \quad v\right|_{e} \in P_{1}(e), \quad \forall e \in \Gamma_{h i}\right\}, \quad i=1,2, \\
\bar{U}=\left\{v\left|v \in L^{2}(\Gamma) ; \quad v\right|_{e}=\text { constant }, \quad \forall e \in \Gamma_{h 2}\right\} .
\end{gathered}
$$

The transfer operator $T: C^{0}\left(\bar{\Omega}_{1}\right) \mapsto U_{1}$ is defined as

$$
(T v)\left(t_{j}^{1}\right)=v\left(t_{j}^{1}\right), \quad j=1,2, \cdots, m_{1}, \quad \forall v \in C^{0}\left(\bar{\Omega}_{1}\right) .
$$

And the $L^{2}$-orthogonal projection operator $S: L^{2}(\Gamma) \mapsto \bar{U}$ is defined by

$$
<S w, v>=<w, v>, \quad \forall w \in L^{2}(\Gamma), v \in \bar{U} .
$$

With these notations and operators, we can define the mortar element space as

$$
W=\left\{v \mid \quad v=\left(v_{1}, v_{2}\right) \in W_{1} \times U_{2} ; \quad S T v_{1}=S v_{2}\right\} .
$$

We assume that $h_{2} \geq h_{1}$ and $m_{2}$ is an odd number. Then the mortar element method can be expressed as:

Find $u_{h}=\left(u_{h 1}, u_{h 2}\right) \in W$ such that

$$
\tilde{b}\left(u_{h}, v\right)=\iint_{\Omega_{1}} f v_{1} d x d y, \quad \forall v=\left(v_{1}, v_{2}\right) \in W,
$$

where $\tilde{b}\left(u_{h}, v\right)=a_{1}\left(u_{h 1}, v_{1}\right)+<K u_{h 2}, v_{2}>_{\Gamma}$ form:

REMARK 2.4. The mortar condition $S T v_{1}=S v_{2}$ can be described in an explicit

$$
v_{2}\left(M_{i}\right)=\frac{1}{\left|e_{i}^{2}\right|} \int_{e_{i}^{2}} T v_{1} d s, \quad i=1,2, \cdots, m_{2}
$$

where $M_{i}$ is the midpoint of the curved segment $e_{i}^{2}$. Since $v_{2}$ is linear function on $e_{i}^{2}$, we have

$$
v_{2}\left(t_{i}^{2}\right)+v_{2}\left(t_{i+1}^{2}\right)=\frac{2}{\left|e_{i}^{2}\right|} \int_{e_{i}^{2}} T v_{1} d s, \quad i=1,2, \cdots, m_{2} .
$$

As $m_{2}$ is an odd number, for any given $v_{1} \in W_{1}$, the mortar condition determines the nodal values $\left\{v_{2}\left(t_{i}^{2}\right)\right\}_{i=1}^{m_{2}}$ uniquely. Also, the explicit form of the mortar condition makes the computation involved in the mortar element method easy to implement and ensures its practical use for practical computation. 
For problem (2.10), we have the following result.

LEMma 2.1. The mortar element problem (2.10) is unisolvable.

Proof. Since $m_{2}$ is an odd number, we can easily find that the mortar space $W$ is a nonempty subspace of the product space $W_{1} \times U_{2}$. Therefore, the unisolvability of the mortar element problem (2.10) follows if we can verify $\tilde{b}(v, v)=0$ with $v=\left(v_{1}, v_{2}\right) \in W$ implies $v_{1}=0$ and $v_{2}=0$.

Now, let us do this. From $a_{1}\left(v_{1}, v_{1}\right)=0$ and $\left.v_{1}\right|_{\partial \Omega}=0$, we know $v_{1}=0$ in $\Omega_{1}$. Then it follows from the mortar condition that $v_{2}\left(t_{i}^{2}\right)=0, i=1,2, \cdots, m_{2}$, i.e. $v_{2}=0$. This completes the proof.

\section{Error estimate}

In this section, we investigate the corresponding error estimate of the mortar element method. In order to do this, some more notations are needed.

Define operator $Q_{1}: H_{*}^{1}\left(\Omega_{1}\right) \mapsto W_{1}$ by

$$
a_{1}\left(Q_{1} v, w\right)=a_{1}(v, w), \quad \forall w \in W_{1} .
$$

For the operator $Q_{1}$, we have the following result(refer to [27]).

Lemma 3.1. The operator $Q_{1}$ satisfies the following inequality

$$
\left\|v-Q_{1} v\right\|_{0, \Omega_{1}}+h_{1}\left\|v-Q_{1} v\right\|_{1, \Omega_{1}} \leq C h_{1}^{2}\|v\|_{2, \Omega_{1}}, \quad \forall v \in H^{2}\left(\Omega_{1}\right) \cap H_{*}^{1}\left(\Omega_{1}\right) .
$$

For any $v \in L^{2}(\Gamma)$, transfer operator $E: L^{2}(\Gamma) \mapsto U_{2}$ is defined by

$$
S(E v)=S v .
$$

Noting that $m_{2}$ is an odd number, we can easily find that the operator $E$ is well defined. And by similar deduction for the explicit form of the mortar condition, we have

$$
(E v)\left(M_{i}\right)=\frac{1}{\left|e_{i}^{2}\right|} \int_{e_{i}^{2}} v(s) d s, \quad i=1,2, \cdots, m_{2} .
$$

Moreover, the operator $E$ has the following properties (refer to [27]).

LEMMA 3.2. The transfer operator E satisfies

$$
\begin{array}{ll}
\|E v\|_{0, \Gamma} \leq C\|v\|_{0, \Gamma}, & \forall v \in L^{2}(\Gamma), \\
\|E v\|_{1, \Gamma} \leq C\|v\|_{1, \Gamma}, & \forall v \in H^{1}(\Gamma) .
\end{array}
$$

With Lemma 3.2, it follows from the Sobolev interpolation theory that

$$
\|E v\|_{\frac{1}{2}, \Gamma} \leq C\|v\|_{\frac{1}{2}, \Gamma}, \quad \forall v \in H^{\frac{1}{2}}(\Gamma) .
$$

After the above preparations, we can define operator $Q: H_{*}^{1}\left(\Omega_{1}\right) \mapsto W$ as

$$
Q v=\left(w_{1}, w_{2}\right), \quad w_{1}=Q_{1} v, w_{2}=E T Q_{1} v .
$$

Noting $S w_{2}=S E T Q_{1} v=S T Q_{1} v=S T w_{1}$, we find that the mortar condition is satisfied under this definition and $Q v \in W$. So, the operator $Q$ is well defined.

For $v=\left(v_{1}, v_{2}\right) \in H^{1}\left(\Omega_{1}\right) \times H^{\frac{1}{2}}(\Gamma)$, we define $\|v\|_{b}^{2}=\left\|v_{1}\right\|_{1, \Omega_{1}}^{2}+\left\|v_{2}\right\|_{\frac{1}{2}, \Gamma^{*}}^{2}$. For the case of $v \in H^{1}\left(\Omega_{1}\right),\|v\|_{b}$ can be defined in the same way by taking $v_{1}=v$ and $v_{2}=\left.v\right|_{\Gamma}$.

With these notations, we first present some property of the operator $Q$. 
THEOREM 3.1. For the operator $Q$, we have

$$
\|v-Q v\|_{b}^{2} \leq C\left(h_{1}^{2}\|v\|_{2, \Omega_{1}}^{2}+h_{2}^{2}\|v\|_{\frac{3}{2}, \Gamma}^{2}\right), \quad \forall v \in H^{2}\left(\Omega_{1}\right) \cap H_{*}^{1}\left(\Omega_{1}\right) .
$$

Proof. From the definition of $Q$ and $\|\cdot\|_{b}$, we have

$$
\|v-Q v\|_{b}^{2}=\left\|v-Q_{1} v\right\|_{1, \Omega_{1}}^{2}+\left\|v_{2}-E T Q_{1} v\right\|_{\frac{1}{2}, \Gamma}^{2}
$$

where $v_{2}=\left.v\right|_{\Gamma}$. And it follows from Lemma 3.1 that

$$
\left\|v-Q_{1} v\right\|_{1, \Omega_{1}}^{2} \leq C h_{1}^{2}\|v\|_{2, \Omega_{1}}^{2} \text {. }
$$

So, we only need to estimate the second term of the right side in (3.2).

Let $I_{h 2} v_{2}$ be the continuous and piecewise linear interpolation function of $v_{2}$ associated with $U_{2}$. Then we have

$$
\left\|v_{2}-I_{h 2} v_{2}\right\|_{\frac{1}{2}, \Gamma} \leq C h_{2}\left\|v_{2}\right\|_{\frac{3}{2}, \Gamma} .
$$

Also, it follows from the fact $T v$ is the continuous and piecewise linear interpolation function of $v$ associated with $U_{1}$ that

$$
\|v-T v\|_{\frac{1}{2}, \Gamma} \leq C h_{1}\|v\|_{\frac{3}{2}, \Gamma} \leq C h_{1}\|v\|_{2, \Omega_{1}} .
$$

Noticing $v_{2}=\left.v\right|_{\Gamma}$ and $E I_{h 2} v_{2}=I_{h 2} v_{2}$, we obtain from (3.1), (3.4) and (3.5) that

$$
\begin{aligned}
\left\|v_{2}-E T Q_{1} v\right\|_{\frac{1}{2}, \Gamma} \leq & \left\|v_{2}-I_{h 2} v_{2}\right\|_{\frac{1}{2}, \Gamma}+\left\|I_{h 2} v_{2}-E T v\right\|_{\frac{1}{2}, \Gamma} \\
& +\left\|E T\left(I-Q_{1}\right) v\right\|_{\frac{1}{2}, \Gamma} \\
\leq & \left\|v_{2}-I_{h 2} v_{2}\right\|_{\frac{1}{2}, \Gamma}+\left\|E\left(I_{h 2} v_{2}-v_{2}\right)\right\|_{\frac{1}{2}, \Gamma} \\
& +\left\|E\left(\left.v\right|_{\Gamma}-T v\right)\right\|_{\frac{1}{2}, \Gamma}+\left\|E T\left(I-Q_{1}\right) v\right\|_{\frac{1}{2}, \Gamma} \\
\leq & C\left(\left\|v_{2}-I_{h 2} v_{2}\right\|_{\frac{1}{2}, \Gamma}+\|v-T v\|_{\frac{1}{2}, \Gamma}+\left\|T\left(I-Q_{1}\right) v\right\|_{\frac{1}{2}, \Gamma}\right) \\
3.6) \leq & C\left(h_{1}\|v\|_{2, \Omega_{1}}+h_{2}\|v\|_{\frac{3}{2}, \Gamma}+\left\|T\left(I-Q_{1}\right) v\right\|_{\frac{1}{2}, \Gamma}\right) .
\end{aligned}
$$

On the other hand, the inverse inequality, Lemma 3.1, Sobolev interpolation theory and error estimates of the interpolation operator $T$ (refer to $[4,10]$ ) imply that, for any $\varepsilon \in\left(0, \frac{1}{2}\right)$,

$$
\begin{aligned}
\left\|T\left(I-Q_{1}\right) v\right\|_{\frac{1}{2}, \Gamma} & \leq C h_{1}^{-\frac{1}{2}}\left\|T\left(I-Q_{1}\right) v\right\|_{0, \Gamma} \\
& \leq C h_{1}^{-\frac{1}{2}}\left(\left\|\left(I-Q_{1}\right) v\right\|_{0, \Gamma}+\left\|(I-T)\left(I-Q_{1}\right) v\right\|_{0, \Gamma}\right) \\
& \leq C h_{1}^{-\frac{1}{2}}\left(\left\|\left(I-Q_{1}\right) v\right\|_{0, \Gamma}+h_{1}^{\frac{1}{2}+\varepsilon}\left\|\left(I-Q_{1}\right) v\right\|_{\frac{1}{2}+\varepsilon, \Gamma}\right) \\
& \leq C\left(h_{1}^{-\frac{1}{2}}\left\|\left(I-Q_{1}\right) v\right\|_{0, \Gamma}+h_{1}^{\varepsilon}\left\|\left(I-Q_{1}\right) v\right\|_{1+\varepsilon, \Omega_{1}}\right) \\
& \leq C\left(h_{1}^{-\frac{1}{2}}\left\|\left(I-Q_{1}\right) v\right\|_{0, \Gamma}+h_{1}\|v\|_{2, \Omega_{1}}\right)
\end{aligned}
$$

Also, from the trace inequality and Lemma 3.1, we have

$$
\left\|\left(I-Q_{1}\right) v\right\|_{0, \Gamma}^{2} \leq C\left\|\left(I-Q_{1}\right) v\right\|_{0, \Omega_{1}}\left\|\left(I-Q_{1}\right) v\right\|_{1, \Omega_{1}} \leq C h_{1}^{3}\|v\|_{2, \Omega_{1}}^{2} .
$$

Thus, using (3.7) and (3.8) gives

$$
\left\|T\left(I-Q_{1}\right) v\right\|_{\frac{1}{2}, \Gamma} \leq C h_{1}\|v\|_{2, \Omega_{1}} .
$$

With (3.2), (3.3), (3.6) and (3.9), we obtain the desired result. This completes the proof.

In order to obtain the corresponding error estimate, we first recall the second Strang Lemma (refer to $[10,14]$ ). 
LEMMA 3.3. Let $u$ be the solution of problem (2.1) and $u_{h}$ be the solution of problem (2.10), then

$$
\left\|u-u_{h}\right\|_{b} \leq c\left(\inf _{v \in W}\|u-v\|_{b}+\sup _{0 \neq v=\left(v_{1}, v_{2}\right) \in W} \frac{\left|\tilde{b}(u, v)-\left(f, v_{1}\right)\right|}{\|v\|_{b}}\right) .
$$

The first term in (3.10) is known as the approximation error and the second term is called the consistency error. So, the error estimate can be obtained if we give out estimates for these items.

With theorem 3.1, the estimate for approximation error can be obtained as follows.

$$
\inf _{v \in W}\|u-v\|_{b} \leq\|u-Q u\|_{b} \leq C\left(h_{1}\|u\|_{2, \Omega_{1}}+h_{2}\|u\|_{\frac{3}{2}, \Gamma}\right) .
$$

For the estimate of the consistency error, we note that

$$
\begin{aligned}
\left|\tilde{b}(u, v)-\left(f, v_{1}\right)\right| & =\left|a_{1}\left(u, v_{1}\right)+<K u, v_{2}>_{\Gamma}-\left(f, v_{1}\right)\right| \\
& =\left|\iint_{\Omega_{1}} \nabla u \cdot \nabla v_{1} d x d y-\int_{\Gamma} \frac{\partial u}{\partial n} v_{2} d s-\left(f, v_{1}\right)\right| \\
& =\left|\iint_{\Omega_{1}}(-\triangle u) v_{1} d x d y+\int_{\Gamma} \frac{\partial u}{\partial n}[v] d s-\left(f, v_{1}\right)\right| \\
& =\left|\int_{\Gamma} \frac{\partial u}{\partial n}[v] d s\right|
\end{aligned}
$$

where $[v]=\left.v_{1}\right|_{\Gamma}-v_{2}$ denotes the jump of $v$ on $\Gamma$. And it follows from the definition of $S$ that

$$
\int_{\Gamma} S \frac{\partial u}{\partial n}\left(T v_{1}-S T v_{1}\right) d s=0
$$

Thus, by using the mortar condition, we get

$$
\begin{aligned}
\left|\int_{\Gamma} \frac{\partial u}{\partial n}[v] d s\right|= & \left|\int_{\Gamma} \frac{\partial u}{\partial n}\left(v_{1}-T v_{1}\right) d s-\int_{\Gamma} \frac{\partial u}{\partial n}\left(v_{2}-S v_{2}\right) d s+\int_{\Gamma} \frac{\partial u}{\partial n}\left(T v_{1}-S v_{2}\right) d s\right| \\
= & \left|\int_{\Gamma} \frac{\partial u}{\partial n}\left(v_{1}-T v_{1}\right) d s-\int_{\Gamma} \frac{\partial u}{\partial n}\left(v_{2}-S v_{2}\right) d s+\int_{\Gamma} \frac{\partial u}{\partial n}\left(T v_{1}-S T v_{1}\right) d s\right| \\
= & \mid \int_{\Gamma} \frac{\partial u}{\partial n}\left(v_{1}-T v_{1}\right) d s-\int_{\Gamma} \frac{\partial u}{\partial n}\left(v_{2}-S v_{2}\right) d s \\
& +\int_{\Gamma}(I-S) \frac{\partial u}{\partial n}\left(T v_{1}-S T v_{1}\right) d s \mid \\
\leq & \left|\int_{\Gamma} \frac{\partial u}{\partial n}\left(v_{1}-T v_{1}\right) d s\right|+\left|\int_{\Gamma} \frac{\partial u}{\partial n}\left(v_{2}-S v_{2}\right) d s\right| \\
& +\left|\int_{\Gamma}(I-S) \frac{\partial u}{\partial n}\left(T v_{1}-S T v_{1}\right) d s\right| \\
\triangleq & I+I I+I I I .
\end{aligned}
$$

Next, we estimate the above three items respectively. We begin by estimating the first term. Noting that $\left.v_{1}\right|_{K_{i}} \in P_{1}\left(K_{i}\right)$ and $T v_{1}$ is the continuous and piecewise linear interpolation of $v_{1}$ on $\Gamma$, we have for any $e_{i}^{1} \in \Gamma_{h 1}$ that

$$
\left\|v_{1}-T v_{1}\right\|_{0, e_{i}^{1}} \leq C h_{1}^{2}\left\|v_{1}\right\|_{2, e_{i}^{1}} \leq C h_{1}^{\frac{5}{2}}\left\|v_{1}\right\|_{2, \infty, e_{i}^{1}}=C h_{1}^{\frac{5}{2}}\left\|v_{1}\right\|_{1, \infty, e_{i}^{1}} \leq C h_{1}^{\frac{3}{2}}\left\|v_{1}\right\|_{1, K_{i}} .
$$


Squaring both sides and summing over all curved elements $K_{i}$ yields

$$
\left\|v_{1}-T v_{1}\right\|_{0, \Gamma}^{2} \leq C h_{1}^{3}\left\|v_{1}\right\|_{1, \Omega_{1}}^{2} .
$$

So, it follows from (3.14) and trace inequality that

$$
\begin{aligned}
I=\left|\int_{\Gamma} \frac{\partial u}{\partial n}\left(v_{1}-T v_{1}\right) d s\right| & \leq C\left\|\frac{\partial u}{\partial n}\right\|_{0, \Gamma}\left\|v_{1}-T v_{1}\right\|_{0, \Gamma} \\
& \leq C h_{1}^{\frac{3}{2}}\left\|\frac{\partial u}{\partial n}\right\|_{0, \Gamma}\left\|v_{1}\right\|_{1, \Omega_{1}} \\
& \leq C h_{1}^{\frac{3}{2}}\|u\|_{2, \Omega_{1}}\left\|v_{1}\right\|_{1, \Omega_{1}} .
\end{aligned}
$$

To estimate the second item $I I$, we recall some property of operator $S$ (refer to [34]).

LEMMA 3.4. For the $L^{2}$ projection operator $S: L^{2}(\Gamma) \mapsto \bar{U}$, the following inequality holds

$$
\|v-S v\|_{0, \Gamma} \leq C h_{2}^{s}\|v\|_{s, \Gamma}, \quad \forall v \in H^{s}(\Gamma), \quad 0 \leq s \leq 1
$$

From Lemma 3.4 and the fact that $\int_{\Gamma} S \frac{\partial u}{\partial n}\left(v_{2}-S v_{2}\right) d s=0$, it follows

$$
\begin{aligned}
I I=\left|\int_{\Gamma} \frac{\partial u}{\partial n}\left(v_{2}-S v_{2}\right) d s\right| & =\left|\int_{\Gamma}(I-S) \frac{\partial u}{\partial n}\left(v_{2}-S v_{2}\right) d s\right| \\
& \leq\left\|(I-S) \frac{\partial u}{\partial n}\right\|_{0, \Gamma}\left\|v_{2}-S v_{2}\right\|_{0, \Gamma} \\
& \leq C h_{2}\left\|\frac{\partial u}{\partial n}\right\|_{\frac{1}{2}, \Gamma}\left\|v_{2}\right\|_{\frac{1}{2}, \Gamma} \\
& \leq C h_{2}\|u\|_{\frac{3}{2}, \Gamma}\left\|v_{2}\right\|_{\frac{1}{2}, \Gamma} .
\end{aligned}
$$

Next, we estimate the third item $I I I$.

Let $\bar{S}_{1}$ be the $L^{2}$-orthogonal projection operator from $L^{2}(\Gamma)$ onto $U_{1}$. The standard argument yields

$$
\left\|w-\bar{S}_{1} w\right\|_{0, \Gamma} \leq C h_{1}\|w\|_{1, \Gamma}, \quad \forall w \in H^{1}(\Gamma),
$$

and for $s=0,1$,

$$
\left\|\bar{S}_{1} w\right\|_{s, \Gamma} \leq C\|w\|_{s, \Gamma}, \quad \forall w \in H^{s}(\Gamma)
$$

which implies

$$
\left\|\bar{S}_{1} w\right\|_{t, \Gamma} \leq C\|w\|_{t, \Gamma}, \quad 0 \leq t \leq 1, \quad \forall w \in H^{t}(\Gamma),
$$

by the Sobolev interpolation theory. Moreover, using the fact

$$
\left\|w-\bar{S}_{1} w\right\|_{0, \Gamma} \leq\|w\|_{0, \Gamma}+\left\|\bar{S}_{1} w\right\|_{0, \Gamma} \leq C\|w\|_{0, \Gamma},
$$

the Sobolev interpolation theory and (3.17) yield

$$
\left\|w-\bar{S}_{1} w\right\|_{0, \Gamma} \leq C h_{1}^{t}\|w\|_{t, \Gamma}, \quad 0 \leq t \leq 1, \quad \forall w \in H^{t}(\Gamma)
$$


With (3.18), (3.14), (3.19), it follows from the inverse inequality and the trace inequality that

$$
\begin{aligned}
\left\|T v_{1}\right\|_{\frac{1}{2}, \Gamma} & \leq\left\|\bar{S}_{1} v_{1}\right\|_{\frac{1}{2}, \Gamma}+\left\|T v_{1}-\bar{S}_{1} v_{1}\right\|_{\frac{1}{2}, \Gamma} \\
& \leq C\left(\left\|v_{1}\right\|_{\frac{1}{2}, \Gamma}+h_{1}^{-\frac{1}{2}}\left\|T v_{1}-\bar{S}_{1} v_{1}\right\|_{0, \Gamma}\right) \\
& \leq C\left[\left\|v_{1}\right\|_{\frac{1}{2}, \Gamma}+h_{1}^{-\frac{1}{2}}\left(\left\|v_{1}-\bar{S}_{1} v_{1}\right\|_{0, \Gamma}+\left\|T v_{1}-v_{1}\right\|_{0, \Gamma}\right)\right] \\
& \leq C\left[\left\|v_{1}\right\|_{\frac{1}{2}, \Gamma}+h_{1}\left\|v_{1}\right\|_{1, \Omega_{1}}\right] \\
& \leq C\left\|v_{1}\right\|_{1, \Omega_{1}}
\end{aligned}
$$

Thus, from Lemma 3.4 and (3.20), we have

$$
\begin{aligned}
I I I & =\left|\int_{\Gamma}(I-S) \frac{\partial u}{\partial n}\left(T v_{1}-S T v_{1}\right) d s\right| \\
& \leq C\left\|(I-S) \frac{\partial u}{\partial n}\right\|_{0, \Gamma}\left\|T v_{1}-S T v_{1}\right\|_{0, \Gamma} \\
& \leq C h_{2}\left\|\frac{\partial u}{\partial n}\right\|_{\frac{1}{2}, \Gamma}\left\|T v_{1}\right\|_{\frac{1}{2}, \Gamma} \\
& \leq C h_{2}\|u\|_{\frac{3}{2}, \Gamma}\left\|v_{1}\right\|_{1, \Omega_{1}} .
\end{aligned}
$$

Then, it follows from (3.12), (3.13), (3.15), (3.16) and (3.21) that

$$
\begin{aligned}
\left|\tilde{b}(u, v)-\left(f, v_{1}\right)\right| \leq & C\left[h_{1}^{\frac{3}{2}}\|u\|_{2, \Omega_{1}}\left\|v_{1}\right\|_{1, \Omega_{1}}\right. \\
& \left.+h_{2}\|u\|_{\frac{3}{2}, \Gamma}\left(\left\|v_{1}\right\|_{1, \Omega_{1}}+\left\|v_{2}\right\|_{\frac{1}{2}, \Gamma}\right)\right] .
\end{aligned}
$$

So, from Lemma 3.3, (3.11) and (3.22), we obtain the following theorem.

THEOREM 3.2. Let $u$ be the solution of problem (2.1) and $u_{h}$ be the solution of problem (2.10), then the following error estimate holds

$$
\left\|u-u_{h}\right\|_{b} \leq C\left(h_{1}\|u\|_{2, \Omega_{1}}+h_{2}\|u\|_{\frac{3}{2}, \Gamma}\right) .
$$

Moreover, if the assumption $\left.u\right|_{\Gamma} \in H^{2}(\Gamma)$ holds, then it follows (see, [36, 39])

$$
\left\|v_{2}-I_{h 2} v_{2}\right\|_{\frac{1}{2}, \Gamma} \leq C h_{2}^{3 / 2}\left\|v_{2}\right\|_{2, \Gamma}
$$

where $v_{2}=\left.u\right|_{\Gamma}$ and $I_{h 2} v_{2}$ is the continuous and piecewise linear interpolation function of $v_{2}$ associated with $U_{2}$. Furthermore, we can get from Lemma 3.4 that

$$
\left\|(I-S) \frac{\partial u}{\partial n}\right\|_{0, \Gamma} \leq C h_{2}\left\|\frac{\partial u}{\partial n}\right\|_{1, \Gamma} \leq C h_{2}\|u\|_{2, \Gamma} .
$$

Using (3.24) for (3.16) and (3.21) and replacing (3.4) with (3.23), we can obtain the following theorem by the same technique in proving Theorem 3.2.

THEOREM 3.3. Let $u$ be the solution of problem (2.1) and $u_{h}$ be the solution of problem (2.10), if $\left.u\right|_{\Gamma} \in H^{2}(\Gamma)$, then the following error estimate holds

$$
\left\|u-u_{h}\right\|_{b} \leq C\left(h_{1}\|u\|_{2, \Omega_{1}}+h_{2}^{3 / 2}\|u\|_{2, \Gamma}\right) .
$$

REMARK 3.1. Since $\Gamma$ is a circle used as artificial boundary in $\Omega^{c}$, it is possible to have $\left.u\right|_{\Gamma} \in H^{2}(\Gamma)$ if $u$ is smooth enough in $\Omega^{c}$. So, for sufficiently smooth $u$, the condition $\left.u\right|_{\Gamma} \in H^{2}(\Gamma)$ is not difficult to satisfy and Theorem 3.3 holds for all these cases. 
REMARK 3.2. An interesting application or byproduct of Theorem 3.3 is that this error estimate can be used to balance the mesh sizes of different sides so as to improve computational efficiency. As we can see, if $h_{1}$ and $h_{2}$ are chosen such that $h_{1}$ and $h_{2}^{3 / 2}$ are of the same order, then it will be more efficient than many other choice of meshes sizes of different sides to obtain the same accuracy. Consequently, many computational cost will be saved by this choice of mesh sizes. So, it is also very useful for practical computation.

REMARK 3.3. In some cases, we may meet difficulties with concave angles in $\Omega_{1}$. Fortunately, many techniques and methods are developed for finite element method to handle this situation successfully (e.g., adaptive finite element method). On the other hand, this situation can also be treated by using boundary element method (see, e.g., [36,39]). So, with only slight modifications, the mortar element method presented here can also be used for this kind of problems.

REMARK 3.4. For solving the discrete problem (2.10), similar D-N alternating method as that presented in $[38,39]$ associated with the coupling of natural boundary element method and finite element method by using matching triangulation on $\Gamma$ can be constructed and used. And the convergence of this D-N alternating method can be obtained in a similar way. Also, many other techniques and preconditioners have been developed and constructed for solving the discrete problem of mortar element method in recent years (see, e.g., [1-3, 9, 13, 22] and references therein). These techniques and preconditioners can also provide many possible approaches for solving (2.10).

REMARK 3.5. Although the mortar element method is developed for Poisson equation over unbounded domain in this paper, the same idea can also be used for more complex unbounded domain problems. Some further research and detailed analysis will be done in our future work. It is hoped that the research toward this direction will bring us much more interesting results.

\section{Numerical results}

In our numerical experiment, we consider the following model problem

$$
\left\{\begin{aligned}
-\triangle u & =f, \quad \text { in } \Omega^{c}, \\
u & =0, \text { on } \partial \Omega,
\end{aligned}\right.
$$

subject to the asymptotic conditions

$$
u(x, y)=\alpha+O(1 / r), \quad|\nabla u(x, y)|=O\left(1 / r^{2}\right), \quad r=\sqrt{x^{2}+y^{2}} \rightarrow \infty,
$$

where $\Omega$ is unit circle disc and $\alpha=1$,

$$
f=\left\{\begin{array}{lc}
\frac{4}{\left(x^{2}+y^{2}\right)^{2}}, & 1<x^{2}+y^{2}<\frac{9}{4}, \\
0, & \frac{9}{4} \leq x^{2}+y^{2} .
\end{array}\right.
$$

We take $\Gamma$ as a circle with radius 2 . In the following tables, $u$ and $u_{h}=\left(u_{h 1}, u_{h 2}\right)$ stand for the exact solution of the model problem and the computational solution respectively. The number of segments $\Gamma$ is divided on the mortar side and the nonmortar side are denoted as $m_{1}$ and $m_{2}$. Notations $h_{1}$ and $h_{2}$ denote the corresponding mesh size of $\mathcal{T}_{1}$ and $\mathcal{T}_{2} . N$ is used to denote the number of unknowns. $\|\cdot\|_{E, \Omega_{1}}$ is a discrete norm equivalent to the norm $\|\cdot\|_{1, \Omega_{1}}$ and $\|\cdot\|_{E, \Gamma}$ is a discrete norm equivalent to the norm $\|\cdot\|_{\frac{1}{2}, \Gamma}$. The i-th element of column "Rat1" stands 
TABle 1. Numerical experiments

\begin{tabular}{cccccccc}
\hline$m_{1}$ & $m_{2}$ & $h_{2} / h_{1}$ & $N$ & $\left\|u-u_{h 1}\right\|_{E, \Omega_{1}}$ & Rat1 & $\left\|u-u_{h 2}\right\|_{E, \Gamma}$ & Rat2 \\
\hline 66 & 33 & 2 & 528 & $5.5259 \mathrm{e}-2$ & - & $1.4551 \mathrm{e}-2$ & - \\
130 & 65 & 2 & 2080 & $2.9372 \mathrm{e}-2$ & 0.5315 & $5.6089 \mathrm{e}-3$ & 0.3855 \\
258 & 129 & 2 & 8256 & $1.4998 \mathrm{e}-2$ & 0.5106 & $2.0505 \mathrm{e}-3$ & 0.3656 \\
514 & 257 & 2 & 32896 & $7.5618 \mathrm{e}-3$ & 0.5042 & $7.3559 \mathrm{e}-4$ & 0.3587 \\
1026 & 513 & 2 & 131328 & $3.7949 \mathrm{e}-3$ & 0.5019 & $2.6184 \mathrm{e}-4$ & 0.3560 \\
\hline
\end{tabular}

TABLE 2. Numerical experiments

\begin{tabular}{cccccccc}
\hline$m_{1}$ & $m_{2}$ & $h_{2} / h_{1}$ & $N$ & $\left\|u-u_{h 1}\right\|_{E, \Omega_{1}}$ & Rat1 & $\left\|u-u_{h 2}\right\|_{E, \Gamma}$ & Rat2 \\
\hline 132 & 33 & 4 & 2112 & $2.9554 \mathrm{e}-2$ & - & $3.9904 \mathrm{e}-3$ & - \\
260 & 65 & 4 & 8320 & $1.5044 \mathrm{e}-2$ & 0.5090 & $1.4544 \mathrm{e}-3$ & 0.3645 \\
516 & 129 & 4 & 33024 & $7.5735 \mathrm{e}-3$ & 0.5034 & $5.2094 \mathrm{e}-4$ & 0.3582 \\
1028 & 257 & 4 & 131584 & $3.7978 \mathrm{e}-3$ & 0.5015 & $1.8529 \mathrm{e}-4$ & 0.3557 \\
\hline
\end{tabular}

TABLE 3. Numerical experiments

\begin{tabular}{cccccccc}
\hline$m_{1}$ & $m_{2}$ & $h_{2} / h_{1}$ & $N$ & $\left\|u-u_{h 1}\right\|_{E, \Omega_{1}}$ & Rat1 & $\left\|u-u_{h 2}\right\|_{E, \Gamma}$ & Rat2 \\
\hline 136 & 17 & 8 & 2176 & $2.9914 \mathrm{e}-2$ & - & $2.8560 \mathrm{e}-3$ & - \\
264 & 33 & 8 & 8448 & $1.5136 \mathrm{e}-2$ & 0.5060 & $1.0347 \mathrm{e}-3$ & 0.3623 \\
520 & 65 & 8 & 33280 & $7.5969 \mathrm{e}-3$ & 0.5019 & $3.6949 \mathrm{e}-4$ & 0.3571 \\
1032 & 129 & 8 & 132096 & $3.8037 \mathrm{e}-3$ & 0.5007 & $1.3122 \mathrm{e}-4$ & 0.3551 \\
\hline
\end{tabular}

for the ratio of the i-th element and the (i-1)-th element of column " $\left\|u-u_{h 1}\right\|_{E, \Omega_{1}} "$ (For example, in Table 1, the second element of column "Rat1" 0.5315 is the ratio of the second element of column " $\left\|u-u_{h 1}\right\|_{E, \Omega_{1}} " 2.9372 e-2$ and the first element of column " $\left\|u-u_{h 1}\right\|_{E, \Omega_{1}} " 5.5259 e-2$, i.e. $\left.0.5315=2.9372 \mathrm{e}-2 / 5.5259 \mathrm{e}-2\right)$. By the same manner, The i-th element of column "Rat2" stands for the ratio of the i-th element and the (i-1)-th element of column " $\left\|u-u_{h 2}\right\|_{E, \Gamma} "$.

With the discrete norm $\|\cdot\|_{E, \Omega_{1}}$ and $\|\cdot\|_{E, \Gamma}$, we can define discrete norm $\|\cdot\|_{E}$ for $x_{h}=\left(x_{h 1}, x_{h 2}\right) \in W$ by

$$
\left\|x_{h}\right\|_{E}^{2}=\left\|x_{h 1}\right\|_{E, \Omega_{1}}^{2}+\left\|x_{h 2}\right\|_{E, \Gamma}^{2} .
$$

Obviously, this discrete norm is equivalent to the norm $\|\cdot\|_{b}$ and

$$
\left\|x_{h}\right\|_{E} \leq\left\|x_{h 1}\right\|_{E, \Omega_{1}}+\left\|x_{h 2}\right\|_{E, \Gamma} .
$$

Only for the convenience of illustration, we make some simple choice and do not use the more efficient choice of mesh sizes as mentioned in Remark 3.2. For practical computation, the more efficient choice of mesh sizes mentioned in Remark 3.2 is recommended. The numerical results are presented in Table 1, Table 2, Table 3 and Table 4.

From all these tables, we can find that when the mesh sizes decrease to the half of the former case, the value of "Rat1" is also about $\frac{1}{2}$ and the value of "Rat2" is 
TABLE 4. Numerical experiments

\begin{tabular}{cccccccc}
\hline$m_{1}$ & $m_{2}$ & $h_{2} / h_{1}$ & $N$ & $\left\|u-u_{h 1}\right\|_{E, \Omega_{1}}$ & Rat1 & $\left\|u-u_{h 2}\right\|_{E, \Gamma}$ & Rat2 \\
\hline 272 & 17 & 16 & 8704 & $1.5321 \mathrm{e}-2$ & - & $7.4053 \mathrm{e}-4$ & - \\
528 & 33 & 16 & 33792 & $7.6435 \mathrm{e}-3$ & 0.4989 & $2.6287 \mathrm{e}-4$ & 0.3550 \\
1040 & 65 & 16 & 133120 & $3.8154 \mathrm{e}-3$ & 0.4992 & $9.3074 \mathrm{e}-5$ & 0.3541 \\
\hline
\end{tabular}

about $\left(\frac{1}{2}\right)^{3 / 2} \approx 0.3536$, which matches our theory well. So, mortar element method performs as what we expect and numerical result supports our theory well.

Acknowledgement. The authors would like to thank two anonymous referees for their comments and suggestions which lead to a great improvement in the presentation of the paper.

\section{References}

1. Y. Achdou and Y. Kuznetsov, Substructing preconditioners for finite element methods on nonmatching grids, East-West J. Numer. Math. 3 (1995), 1-28.

2. Y. Achdou, Y. Kuznetsov, and O. Pironneau, Substructing preconditioner for the $q_{1}$ mortar element method, Numer. Math. 71 (1995), 419-449.

3. Y. Achdou, Y. Maday, and O. Widlund, Iterative substructing preconditioners for mortar element methods in two dimensions, SIAM J. Numer. Anal. 36 (1999), 551-580.

4. I. Babuska and A. Aziz, Survey lectures on the mathematical foundations of the finite element method, The Mathematical Foundations of thr Finite Element Method with Applications to Partial Differential Equations (A. Aziz, ed.), Academic Press, New York, 1972.

5. F. Ben Belgacem, The mortar finite element method with lagrange multipliers, Numer. Math. 84 (1999), 2173-2197.

6. F. Ben Belgacem, A. Buffa, and Y. Maday, The mortar finite element method for $3 D$ Maxwell equations: first results, SIAM J. Numer. Anal. 39 (2001), 880-901.

7. F. Ben Belgacem and Y. Maday, The mortar element method for three-dimensional finite element, RAIRO Model. Math. Anal. Numer. 31 (1997), 289-302.

8. C. Bernardi, Y. Maday, and A. Patera, A new nonconforming approach to domain decomposition: The mortar element method, Nonlinear partial differential equations and their applications (H. Brezis and J. L. Lions, eds.), Pitman Research Notes in Mathmatics, no. 299, Longman Scientific Technical, 1994.

9. D. Braess, W. Dehmen, and C. Wieners, A multigrid algorithm for the mortar finite element method, SIAM J. Numer. Anal. 37 (2000), 48-69.

10. S. Brenner and L. Scott, The mathematical theory of finite element method, Springer, New York, 1994.

11. F. Brezzi and C. Johnson, On the coupling of boundary integral and finite element methods, Calcolo 16 (1979), 189-201.

12. U. Brink and E. Stephan, Convergence rates for the coupling of FEM and BEM, IMA J. Numer. Anal. 16 (1996), 93-110.

13. M. Casarin and O. Widlund, A hierarchical preconditioner for the mortar finite element method, ETNA 4 (1996), 75-88.

14. P. Ciarlet, The finite element method for elliptic problems, North-Holland Publishers, Amsterdam, 1978.

15. M. Costabel, Symmetric methods for the coupling of finite elements and boundary elements, Boundary Elements IV, vol. 1, Comput. Mechanics Publications, Southampton, 1987, pp. 411420.

16. R. Ewing, R. Lazarov, T. Lin, and Y. Lin, Mortar finite volume element approximations of second order elliptic problem, East-West J. Numer. Math. 8 (2000), 93-110. 
17. K. Feng, Differential versus integral equations and finite versus infinite elements, Math. Numer. Sinica 2 (1980), no. 1, 100-105.

18. $\quad$ Finite element method and natural boundary reduction, Proceedings of the International Congress of Mathematicians, Warszawa, 1983, pp. 1439-1453.

19. K. Feng and D. Yu, Canonical integral equation of elliptic boundary value problems and their numerical solution, Proc. of China-France Symp. on FEM (Beijing), Beijing, 1982, Science Press, 1983, pp. 221-252.

20. G. Gatica and G. Hsiao, The coupling of boundary element and finite element methods for a nonlinear exterior boundary value problem, Zeitschr. Anal. Anw. 28 (1989), 377-387.

21. D. Givoli and J. Keller, A finite element method for large domains, Comput. Methods Appl. Mech. Engrg. 76 (1989), no. 1, 41-66.

22. J. Gopalakrishnan and J. Pasciak, Multigrid for the mortar finite element method, SIAM J. Numer. Anal. 37 (2000), 1029-1052.

23. H. Han, A new class of variational formulations for the coupling of finite element and boundary element methods, J. Comp. Math. 8 (1990), 223-232.

24. H. Han and X. Wu, Approximation of infinite boundary condition and its application to finite element methods, J. Comput. Math. 3 (1985), no. 2, 179-192.

25. H. Han and L. Ying, Large element and the local finite element, Acta Math. Appl. Sinica 3 (1980), no. 3, 237-249.

26. G. Hsiao, The coupling of BEM and FEM - a brief review, Boundary Element X 1 (1988), 431-446.

27. J. Huang and J. Zou, A mortar element method for elliptic problems with discontinuous coefficients, IMA J. Numer. Anal. 22 (2002), 549-576.

28. C. Johnson and J. Nédélec, On the coupling of boundary integral and finite element methods, Math. Comp. 35 (1980), 1063-1079.

29. J. Keller and D. Givoli, Exact nonreflecting boundary conditions, J. Comput. Phys. 82 (1989), no. $1,172-192$.

30. L. Marcinkowski, The mortar element method with locally nonconforming elements, BIT 39 (1999), 716-739.

31. P. Seshaiyer and M. Suri, Uniform $h p$ convergence results for the mortar finite element method, Math. Comp. 69 (2000), 521-546.

32. W. Wendland, On asymptotic error estimates for the combined BEM and FEM, Innovative Numerical Methods in Engrg. 88 (1986), 55-70.

33. B. Wohlmuth, A mortar finite element method using dual spaces for the Lagrange multiplier, SIAM J. Numer. Anal. 38 (2000), 989-1012.

34. J. Xu, Theory of multilevel methods, Ph.D. thesis, Cornell University, 1989.

35. D. Yu, Canonical integral equations of biharmonic elliptic boundary value problems, Math. Numer. Sinica, 4 (1982), no. 3, 330-336.

36. - The mathematical theory of the natural boundary element method, Science Press, Beijing, 1993, (in Chinese).

37. The relation between the Steklov-Poincaré operator, the natural integral operator and Green functions, Chinese J. Numer. Math. Appl. 17 (1995), no. 4, 95-106.

38. - Discretization of non-overlapping domain decomposition method for unbounded domains and its convergence, Chinese J. Numer. Math. Appl. 18 (1996), no. 4, 93-102.

39. _ The natural boundary integral method and its applications, Kluwer Academic Publisher/Science Press, Beijing, 2002.

40. O. Zienkiewicz, D. Kelly, and P. Bettess, The coupling of the finite element method and boundary solution procedures, Int. J. for Numer. Methods in Engrg. 11 (1977), 355-375.

Institute of Computational Mathematics and Scientific/Engineering Computing, Academy of Mathematics and Systems Science, Chinese Academy of Science, Beijing, 100080, People's Republic of China

E-mail address: szhang@lsec.cc.ac.cn

Institute of Computational Mathematics and Scientific/Engineering Computing, Academy of Mathematics and Systems Science, Chinese Academy of Science, Beijing, 100080, People's Republic of China

E-mail address: ydh@lsec.cc.ac.cn 